\title{
Correction to: Blast Disease of Cereal Crops: Evolution and Adaptation in Context of Climate Change
}

S. Chandra Nayaka, Rajashekara Hosahatti, Ganesan Prakash,

C. Tara Satyavathi, and Rajan Sharma

\section{Correction to:}

S. C. Nayaka et al. (eds.), Blast Disease of Cereal Crops, Fungal Biology, https://doi.org/10.1007/978-3-030-60585-8

This book was inadvertently published with incorrect co-editor name as "Ganesh" instead of "Ganesan". This is now corrected in all the renditions. 